\title{
Analysing globular cluster observations
}

\section{Models and analysis tools for Lick/IDS indices}

\author{
T. Lilly ${ }^{1}$ and U. Fritze - v. Alvensleben ${ }^{2}$ \\ 1 Institut für Astrophysik, Friedrich-Hund-Platz 1, 37077 Göttingen, Germany \\ e-mail: tlilly@astro.physik.uni-goettingen.de \\ ${ }^{2}$ Centre for Astrophysics Research, STRI, University of Hertfordshire, College Lane, Hatfield AL10 9AB, UK \\ e-mail: ufritze@star.herts.ac.uk
}

Received 21 January 2005 / Accepted 16 May 2006

\begin{abstract}
We have extended our evolutionary synthesis code, GALEV, to include Lick/IDS absorption-line indices for both simple and composite stellar population models (star clusters and galaxies), using polynomial fitting functions. We present a mathematically advanced Lick index analysis tool (LINO) for the determination of the ages and metallicities of globular clusters (CGs). An extensive grid of GALEV models for the evolution of star clusters at various metallicities over a Hubble time is compared to observed sets of Lick indices of varying completeness and precision. A dedicated $\chi^{2}$-minimisation procedure selects the best model including $1 \sigma$ uncertainties on age and metallicity. We discuss the age and metallicity sensitivities of individual indices and show that these sensitivities themselves depend on age and metallicity; thus, we extend Worthey's (1994) concept of a "metallicity sensitivity parameter" for an old stellar population at solar metallicity to younger clusters of different metallicities. We find that indices at low metallicity are generally more age sensitive than at high metallicity. Our aim is to provide a robust and reliable tool for the interpretation of star-cluster spectra becoming available from $10 \mathrm{~m}$ class telescopes in a large variety of galaxies - metal-rich \& metal-poor, starburst, post-burst, and dynamically young. We test our analysis tool using observations from various authors for Galactic and M31 GCs, for which reliable age and metallicity determinations are available in the literature, and discuss to what extent the observational availability of various subsets of Lick indices affects the results. For M31 GCs, we discuss the influence of non-solar abundance ratios on our results. All models are accessible from our website, http://www.astro.physik.uni-goettingen.de/ galev/
\end{abstract}

Key words. globular clusters: general - galaxies: stellar content - galaxies: evolution - galaxies: elliptical and lenticular, cD techniques: spectroscopic

\section{Introduction}

In order to understand the formation and evolution of galaxies, one of the essential issues is to reveal their star formation histories (SFHs). Unfortunately, most galaxies are observable only in integrated light, so that SFH determinations using the most reliable CMD approach are only possible for a very limited sample of nearby galaxies. However, the age and metallicity distributions of star cluster and globular cluster (GC) systems can provide important clues to the formation and evolutionary history of their parent galaxies. For example, the violent formation history of elliptical galaxies, as predicted from hierarchical or merger scenarios, is, in fact, obtained more directly from the age and metallicity distributions of their GC systems than from their integrated spectra, which are always dominated by stars originating in the last major star-formation episode. By means of evolutionary synthesis models, for example, we can show that, when using the integrated light of a galaxy's (composite) stellar content alone, it is impossible to date (and actually to identify) even a very strong starburst if this event took place more than two or three Gyrs ago (Lilly 2003; Lilly \& Fritze - von Alvensleben 2005). Therefore, it is an important first step towards understanding the formation and evolution of galaxies to constrain the age and metallicity distributions of their star cluster systems (for recent reviews see, for example, Kissler-Patig 2000; and Zepf 1999, 2002), as well as of their stars (see, e.g., Harris et al. 1999; Harris \& Harris 2000, 2002). Star clusters can be observed one-by-one to fairly high precision in galaxies out to Virgo cluster distances, even on bright and variable galaxy backgrounds, in terms of both multi-band imaging and intermediateresolution spectroscopy. For young star cluster systems, we have shown that the age and metallicity distributions can be obtained from a comparison of multi-band imaging data with a grid of model SEDs using the SED analysis tool AnalySED (Anders et al. 2004).

Our aim is to extend the analysis of star cluster age and metallicity distributions in terms of parent galaxy formation histories and scenarios to intermediate age and old star cluster systems. However, for all colours the evolution slows down considerably at ages older than about 8 Gyr. Even with several passbands and a long wavelength basis, the results are extremely uncertain for old GCs; colours - even when combining optical and near infrared - do not allow the age-metallicity degeneracy to be completely disentangled (cf. Anders et al. 2004). Absorption-line indices, on the other hand, are a promising tool for independent and more precise constraints on ages and metallicities. Therefore, we present a grid of new evolutionary synthesis models for star clusters, including Lick/IDS indices, to complement the broad band colours and spectra of our previous models, and a Lick index analysis tool LINO meant to 
complement our SED analysis tool. With these two analysis tools, we now possess reasonable procedures for interpreting both broad-band colour and spectral index observations.

In an earlier study, we already incorporated a subset of Lick indices into our evolutionary synthesis code GALEV (Kurth et al. 1999). However, since then the input physics for the code has changed considerably; instead of the older tracks, we are now using up-to-date Padova isochrones, which include the thermally pulsing asymptotic giant branch (TP-AGB) phase of stellar evolution (see Schulz et al. 2002). In this work, we present the integration of the full set of Lick indices into our code. Now, our GALEV models consistently describe the time evolution of spectra, broad-band colours, emission lines, and Lick indices for both globular clusters (treated as single-age single-metallicity, i.e. "simple" stellar populations, SSPs) and galaxies (composite stellar populations, CSPs), using the same input physics for all models (for an exhaustive description of GALEV and its possibilities, as well as for recent extensions of the code and its input physics, see Schulz et al. 2002; Anders \& Fritze- v. Alvensleben 2003; and Bicker et al. 2004).

A recent publication (Proctor et al. 2004) also presented an analysis tool for Lick indices using an $\chi^{2}$-approach. However, they do not provide any confidence intervals for their best-fitting models. In this respect, our new tool extends their approach. A drawback of our models is that, at the present stage, they do not account for variations in $\alpha$-enhancement, as Proctor et al. (2004) do. However, our analysis tool LINO is easily applicable to any available set of absortion line indices.

In Sect. 2, we recall the basic definitions of Lick indices and describe how we synthesize them in our models; we also address non-solar abundance ratios. Some examples of SSP model indices are presented and briefly confronted with observations. In Sect. 3, Worthey's (1994) "metallicity sensitivity parameter" is discussed and extended from old stellar populations to stellar populations of all ages. Section 4 describes and tests our new Lick index analysis tool; Galactic and M 31 globular cluster observations are analysed and compared with results (taken from the literature) from reliable CMD analysis, and from index analyses using models with varying $\alpha$-enhancements, respectively. Section 5 summarises the results and provides an outlook.

\section{Models and input physics}

In this section, we give an overview of our GALEV models and describe how we synthesize Lick indices. We address the impact of non-solar abundance ratios on our results and compare some examples of SSP model indices with observations.

\subsection{Evolutionary synthesis of Lick indices}

Lick indices are relatively broad spectral features, and robust to measure. They are named after the most prominent absorption line in the respective index's passband. However, this does not necessarily mean that a certain index's strength is exclusively, or even dominantly, due to line(s) of this element (see, e.g., Tripicco \& Bell 1995). Beyond the fact that more than one line can be present in the index's passband, strong lines in the pseudo-continua can also affect the index strength. Most indices are given in units of their equivalent width $(E W)$ measured in $\AA$,

$E W[\AA]=\int_{\lambda_{1}}^{\lambda_{2}} \frac{F_{\mathrm{C}}(\lambda)-F_{\mathrm{I}}(\lambda)}{F_{\mathrm{C}}(\lambda)} \mathrm{d} \lambda$, whereas index strengths of broad molecular lines are given in magnitudes:

$I[\mathrm{mag}]=-2.5 \log \left[\left(\frac{1}{\lambda_{1}-\lambda_{2}}\right) \int_{\lambda_{1}}^{\lambda_{2}} \frac{F_{\mathrm{I}}(\lambda)}{F_{\mathrm{C}}(\lambda)} \mathrm{d} \lambda\right]$.

Here, $F_{\mathrm{I}}(\lambda)$ is the flux in the index covering the wavelength range between $\lambda_{1}$ and $\lambda_{2}$, and $F_{\mathrm{C}}(\lambda)$ is the continuum flux defined by two "pseudo-continua" flanking the central index passband.

There are currently 25 Lick indices, all within the optical wavelength range: $\mathrm{H} \delta_{A}, \mathrm{H} \gamma_{A}, \mathrm{H} \delta_{F}, \mathrm{H} \gamma_{F}, \mathrm{CN}_{1}, \mathrm{CN}_{2}, \mathrm{Ca} 4227$, G4300, Fe4383, Ca4455, Fe4531, Fe4668, H $\beta, F e 5015, \mathrm{Mg}_{1}$, $\mathrm{Mg}_{2}, \mathrm{Mg} b, \mathrm{Fe} 5270, \mathrm{Fe} 5335, \mathrm{Fe} 4506, \mathrm{Fe} 5709$, Fe5782, Na D, $\mathrm{TiO}_{1}$, and $\mathrm{TiO}_{2}$. For a full description and all index definitions, see Trager et al. (1998) and references therein.

As the basis for our evolutionary synthesis models, we employ the polynomial fitting functions of Worthey et al. (1994) and Worthey \& Ottaviani (1997), which give Lick index strengths of individual stars as a function of their effective temperature $T_{\text {eff }}$, surface gravity $g$, and metallicity $[\mathrm{Fe} / \mathrm{H}]$. Worthey et al. have calibrated their fitting functions empirically using solar-neighbourhood stars.

Model uncertainties are calculated as follows (Worthey 2004):

$\sigma_{\text {model }}=\frac{\sigma_{\text {star }} \times \mathrm{RMS}_{\mathrm{fit}}}{\sqrt{N}}$

with $\sigma_{\text {star }}$ the typical rms error per observation for the calibration stars and $\mathrm{RMS}_{\mathrm{fit}}$ the residual rms of the fitting functions; both values are given in Worthey et al. (1994) and Worthey \& Ottaviani (1997). Here, $N$ is the number of stars in the "neighbourhood" of the fitting functions in the $T_{\text {eff }}, g,[\mathrm{Fe} / \mathrm{H}]$ space, which is typically of the order of 25 . Note that this approach is only an approximation; the real model error is most likely a strong function of $T_{\text {eff }}, g$, and $[\mathrm{Fe} / \mathrm{H}]$.

Other input physics of our models include the theoretical spectral library from Lejeune et al. (1997, 1998), as well as theoretical isochrones from the Padova group for $Z=0.0004$, 0.004, 0.008, 0.02, and 0.05 (cf. Bertelli et al. 1994), and for $Z=0.0001$ (cf. Girardi et al. 1996). Recent versions of these isochrones include the TP-AGB phase of stellar evolution (not presented in the referenced papers), which is important for intermediate age stellar populations (cf. Schulz et al. 2002). We assume a standard Salpeter (1955) initial mass function (IMF) from 0.15 to about $70 M_{\odot}$; lowest mass stars $\left(M_{\odot}<0.6\right)$ are taken from Chabrier \& Baraffe (1997; cf. Schulz et al. 2002, for details). Throughout this paper, we identify the metallicity $Z$ with $[\mathrm{Fe} / \mathrm{H}]$ and define $[\mathrm{Fe} / \mathrm{H}]:=\log \left(Z / Z_{\odot}\right)$.

To calculate the time evolution of Lick indices for SSP or galaxy models, we follow four steps:

1. We use the values for $T_{\mathrm{eff}}, g$, and $[\mathrm{Fe} / \mathrm{H}]$ given (directly or indirectly) by the isochrones to compute the index strength $E W_{\text {star }}$ or $I_{\text {star }}$ for each star along the isochrones.

2. A spectrum is assigned to each star on a given isochrone and used to compute its continuum flux $F_{\mathrm{C}}{ }^{1}$.

3. For each isochrone, the index strengths are integrated over all stellar masses $m$ (after transformation of the index strengths into fluxes), weighted by the IMF (using a weighting factor $w$ )

$$
E W_{\mathrm{SSP}}=\left(\lambda_{2}-\lambda_{1}\right) \cdot\left(1-\frac{\sum_{m}\left(F_{\mathrm{I}} \cdot w\right)}{\sum_{m}\left(F_{\mathrm{C}} \cdot w\right)}\right),
$$

1 In view of the resolution of our spectral library, these values are not very accurate; however, since $F_{\mathrm{C}}$ is merely an additional weighting factor for the integration routine, this does not affect the final results. 

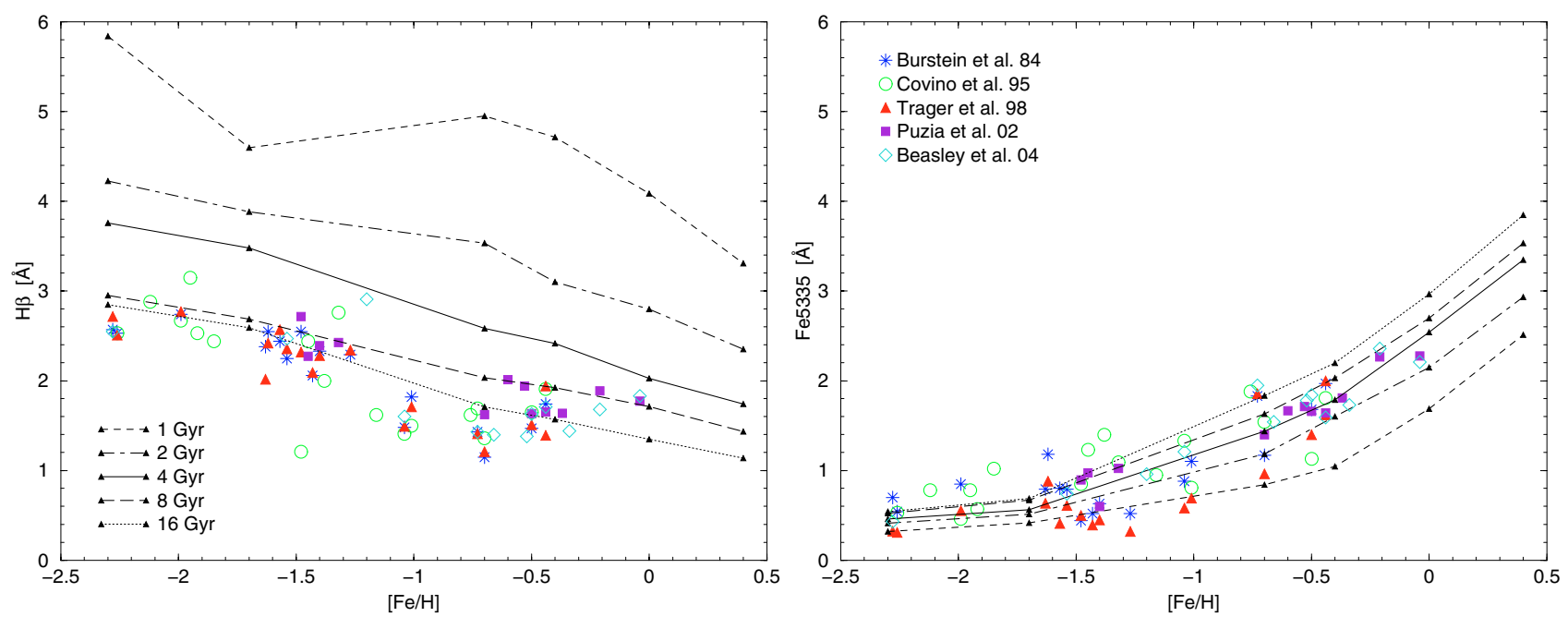

Fig. 1. Indices $\mathrm{H} \beta$ (left) and Fe5335 (right) versus metallicity for 5 different ages. Also shown are Galactic GC observations from various authors as indicated in the right-hand panel; GC metallicities are taken from Harris (1996, revision Feb. 2003). A typical measurement error is about $0.2 \AA$.

where $F_{\mathrm{I}}$ is a function of $E W_{\text {star }}$ and $F_{\mathrm{C}}$ :

$$
F_{\mathrm{I}}=F_{\mathrm{C}} \cdot\left(1-\frac{E W_{\text {star }}}{\lambda_{2}-\lambda_{1}}\right) .
$$

The result of these 3 steps is a grid of SSP models for all available isochrones, i.e., a grid consisting of the 6 metallicities given above and 50 ages between $4 \mathrm{Myr}$ and $20 \mathrm{Gyr}$.

4. For each time step in the computation of a stellar population model, our evolutionary synthesis code GALEV gives the contribution of each isochrone to the total population.

To obtain galaxy model indices (or a better age resolution for SSP models), we integrate our grid of SSP models using Eqs. (4) and (5) again, but with $w$ the isochrone contribution as a new weighting factor (now doing the summation over all isochrones instead of all masses), $F_{\mathrm{C}}$ the integrated continuum flux level for each isochrone, and using $E W_{\text {SSP }}$ instead of $E W_{\text {star }}$.

This way, we computed a large grid of SSP models, consisting of 6 metallicities and 4000 ages from 4 Myr to 16 Gyr in steps of $4 \mathrm{Myr}$; each point of the model grid consists of all the $25 \mathrm{Lick}$ indices currently available.

\subsection{Non-solar abundance ratios}

Abundance ratios reflect the relation between the characteristic time scale of star formation and the time scales for the release of, e.g., SNe II products ( $\mathrm{Mg}$ and other $\alpha$-elements), $\mathrm{SNe} \mathrm{Ia}$ products $(\mathrm{Fe})$, or nucleosynthetic products from intermediatemass stars (N). Galaxies with different SFHs will hence be characterised by different distributions of stellar abundances ratios. This means that the Galactic relation between abundance ratios and metallicity (Edvardsson et al. 1993; Pagel \& Tautvaišienè 1995 ) is not necessarily valid for galaxies of different types and formation histories. Empirical index calibrations based on Galactic stars, like the fitting functions from Worthey et al. (1994) and Worthey \& Ottaviani (1997) applied in this work, are based on the implicit inclusion of the Galactic relation between abundance ratios and metallicity.
A lot of work has been done in past years to study the impact of $\alpha$-enhancement on stellar population models and their applications; e.g., based mainly on the work of Tripicco \& Bell (1995) and Trager et al. (2000a), Thomas et al. (2003, 2004) present SSP models of Lick indices with variable abundance ratios that are corrected for the bias mentioned above, providing for the first time well-defined $[\alpha / \mathrm{Fe}]$ ratios at all metallicities. The impact of these new models on age and metallicity estimates of early type galaxies is investigated in detail by Maraston et al. (2003), Thomas \& Maraston (2003), Thomas et al. (2004), Trager et al. (2000a,b), among others.

However, since our purpose is to present consistently computed models for spectra, colours, emission lines, and Lick indices for both SSPs and CSPs, a consistent attempt to allow our evolutionary synthesis code GALEV to account for arbitrary abundance ratios would have to be based on stellar evolutionary tracks or isochrones, detailed nucleosynthetic stellar yields, and model atmospheres for various abundance ratios. Since both consistent and complete datasets of this kind are not yet available (though first sets of evolutionary tracks for stars with enhanced $[\alpha / \mathrm{Fe}]$ ratios were presented by Salasnich et al. 2000; and Kim et al. 2002), our models at the present stage do not explicitly allow for variations in $\alpha$-enhancement. This is an important caveat to be kept in mind for the interpretation of extragalactic GC populations. We think that the extensive studies of non-solar abundance ratios cited above will allow us to estimate the impact of this caveat on our results.

However, in Sect. 4.3 we show that our method is robust enough to give very good age and metallicity determinations for GCs even without using $\alpha$-enhanced models.

\subsection{SSP model indices: some examples}

In Figs. 1 and 2, we show the time evolution and metallicity dependence of the indices $\mathrm{H} \beta$ and Fe5335 in our new SSP models, and compare them with index measurements of Galactic GCs that are plotted against reliable age and metallicity estimates, respectively.

In particular, in Fig. 1 we compare SSP models for five ages between 1 and $16 \mathrm{Gyr}$ with Galactic GC observations by Burstein et al. (1984, 17 clusters), Covino et al. (1995, 

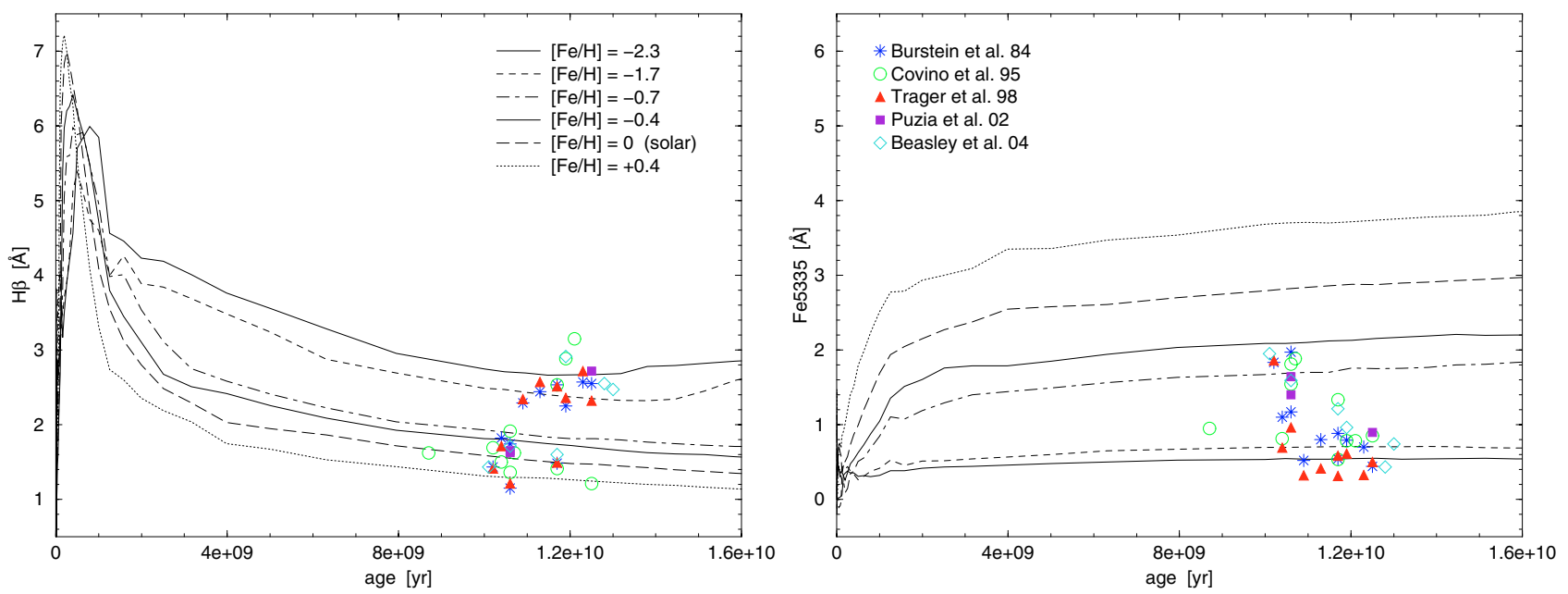

Fig. 2. Indices $\mathrm{H} \beta$ (left) and Fe5335 (right) versus age for 6 different metallicities. Also shown are Galactic GC observations from various authors as indicated in the right-hand panel; GC age determinations are taken from Salaris \& Weiss (2002).

17 clusters), Trager et al. (1998, 18 clusters), Puzia et al. (2002, 12 clusters), and Beasley et al. (2004, 12 clusters). Note that some clusters were observed repeatedly, so more than one data point in the figure can refer to the same cluster. The metallicities are taken from Harris (1996, revision Feb. 2003). In Fig. 2 we show the time evolution of the model indices for all six metallicities, and compare them with Galactic GC observations (taken from the same references as in Fig. 1). The GC age determinations are based on CMD fits and taken from Salaris \& Weiss $(2002)^{2}$. Over the range of Galactic GC ages and metallicities (i.e., ages older than about $8 \mathrm{Gyr}$ and metallicities lower than solar in most cases), a sufficient agreement is observed between models and data in the sense that the data lie within the range of the model grid; we also checked this for other indices (not plotted).

However, the plots also demonstrate how difficult it would be to interpret the indices in terms of classical index-index plots. Actually, Fig. 2 seems to show apparent inconsistencies, so some clusters in Fig. 2 have metallicities up to $[\mathrm{Fe} / \mathrm{H}]=+0.4$ when compared with models for the age-sensitive index $\mathrm{H} \beta$, whereas, when compared with models for the metallicity-sensitive Fe5335 index, all clusters have metallicities lower than $[\mathrm{Fe} / \mathrm{H}]=-0.4$. We cannot decide at this point to what degree these inconsistencies are due to problems in the models or the calibrations they are based on or due to badly calibrated observations; however, our new Lick index analysis tool nevertheless gives surprisingly robust age and, particularly, metallicity determinations for the same set of cluster observations (cf. Sect. 4.2).

\section{Index sensitivities}

It is well known that different indices have varying sensitivities to age and/or metallicity. To quantify this, Worthey (1994) introduced a "metallicity sensitivity parameter" that gives a hint of how sensitive a given index is with respect to changes in age and metallicity. This parameter is defined as the ratio of the percentage change in $Z$ to the percentage change in age (so influences of

${ }^{2}$ Note that they only cover a subsample of the observations shown in Fig. 1: 11 clusters of the Burstein et al. (1984) sample, 10 of the Covino et al. (1995) sample, 10 of the Trager et al. (1998) sample, only 3 of the Puzia et al. (2002) sample, and 6 clusters of the Beasley et al. (2004) sample. possible age-metallicity degeneracies are implicitely included), with large numbers indicating greater metallicity sensitivity:

$S=\left(\frac{\Delta I_{Z}}{\Delta Z / Z}\right) /\left(\frac{\Delta I_{\text {age }}}{\Delta \text { age } / \text { age }}\right)$.

Using his SSP models, Worthey (1994) chose a 12 Gyr solar metallicity $(Z=0.017)$ model as the zero point for the sensitivity parameters, the $\Delta$ 's referring to "neighbouring" models, in this case models with age $=8 / 17 \mathrm{Gyr}$ (i.e, $\Delta$ age $=4 / 5 \mathrm{Gyr}$ ) and $Z=0.01 / 0.03$ (i.e., $\Delta Z=0.007 / 0.013)^{3}$; the main numerator/denominator in Eq. (6) is averaged using both $\Delta$ 's before computing the fraction.

In Table 1, we reprint the metallicity sensitivity parameters given by Worthey (1994) and Worthey \& Ottaviani (1997), and compare them with parameters computed using our own models. Extending Worthey's approach, we computed parameters for four different combinations of zero points, using high $(Z=0.02)$ and low $(Z=0.0004)$ metallicities, along with high $(12 \mathrm{Gyr})$ and intermediate (4 Gyr) ages.

Worthey's parameters are reproduced relatively well by models with a similar combination of zero points, i.e. for old (12 Gyr) and solar metallicity SSPs. However, S is not totally independent of the $\Delta Z$ and $\Delta$ age chosen, since it can be very sensitive to the exact evolution of the model index. This occurs mainly in age-metallicity space regions where the slope of the index does not evolve very smoothly. For example, a very high value of S can also mean that, due to a small "bump" in the time evolution of the index, $\Delta I_{\text {age }}$ is near zero; in this case, $S$ is worthless.

The two zero points for both age and metallicity and their "neighbouring models" we use for the computations are given in Table 2; for both age zero points, we chose two sets of neighbouring models and averaged the final parameters. To check the reliability of our results, we also computed parameters for values of age and $\Delta$ age not given in the table. If the results for different $\Delta$ age's (or slightly different zero points) differ strongly, we classify the parameter as uncertain (indicated by brackets in Table 1). The "ranking" of indices in terms of sensitivity is, with some exceptions, unaffected by changes in the zero points.

3 Ideally, $S$ should be relatively independent of the exact values of the $\Delta Z$ and $\Delta$ age chosen, as long as they are not too large. 
Table 1. Metallicity sensitivity parameters for different zero points. Low numbers indicate high age sensitivity. Values given in brackets are not reliable (see text).

\begin{tabular}{cccccc}
\hline \hline & WORTHEY & \multicolumn{2}{c}{ GALEV $12 \mathrm{Gyr}$} & \multicolumn{2}{c}{ GALEV 4 Gyr } \\
& & $Z=0.02$ & 0.0004 & 0.02 & 0.0004 \\
\hline $\mathrm{CN}_{1}$ & 1.9 & 1.5 & 1.1 & 1.1 & 0.1 \\
$\mathrm{CN}_{2}$ & 2.1 & 1.5 & 0.2 & 1.2 & 0.1 \\
$\mathrm{Ca} 4227$ & 1.5 & 1.1 & $(0.4)$ & 1.0 & 0.1 \\
$\mathrm{G} 4300$ & 1.0 & 1.0 & 0.2 & 0.8 & 0.1 \\
$\mathrm{Fe} 4383$ & 1.9 & 1.9 & 0.3 & 1.3 & 0.2 \\
$\mathrm{Ca} 4455$ & 2.0 & 1.7 & 0.7 & 1.5 & 0.3 \\
$\mathrm{Fe} 4531$ & 1.9 & 1.7 & 0.6 & 1.4 & 0.2 \\
$\mathrm{Fe} 4668$ & 4.9 & $(3.5)$ & $(0.9)$ & 2.4 & 0.9 \\
$\mathrm{H} \beta$ & 0.6 & 0.6 & 0.1 & 0.5 & 0.1 \\
$\mathrm{Fe} 5015$ & 4.0 & $(2.3)$ & $(1.3)$ & 2.1 & 0.4 \\
$\mathrm{Mg} g_{1}$ & 1.8 & 1.7 & $(2.2)$ & 1.4 & 2.0 \\
$\mathrm{Mg}{ }_{2}$ & 1.8 & 1.5 & $(1.8)$ & 1.2 & 0.5 \\
$\mathrm{Mg} b$ & 1.7 & 1.4 & $(0.7)$ & 1.0 & 0.3 \\
$\mathrm{Fe} 5270$ & 2.3 & 2.0 & $(0.7)$ & 1.6 & 0.3 \\
$\mathrm{Fe} 5335$ & 2.8 & 2.7 & $(1.3)$ & 2.0 & 0.4 \\
$\mathrm{Fe} 5406$ & 2.5 & $(2.6)$ & $(2.3)$ & 1.8 & 0.6 \\
$\mathrm{Fe} 5709$ & 6.5 & $(8.5)$ & $(1.7)$ & 2.6 & $(1.2)$ \\
$\mathrm{Fe} 5782$ & 5.1 & $(5.9)$ & $(1.4)$ & 2.5 & $(1.0)$ \\
$\mathrm{Na} \mathrm{D}$ & 2.1 & 1.9 & $(1.2)$ & 1.9 & 0.6 \\
$\mathrm{TiO}_{1}$ & 1.5 & 0.9 & 0.7 & $(1.4)$ & $(5.5)$ \\
$\mathrm{TiO}{ }_{2}$ & 2.5 & 1.3 & 0.9 & $(1.6)$ & $(8.6)$ \\
$\mathrm{H} \delta_{A}$ & 1.1 & 1.0 & 0.3 & 0.8 & 0.1 \\
$\mathrm{H} \gamma_{A}$ & 1.0 & 1.0 & 0.2 & 0.8 & 0.1 \\
$\mathrm{H} \delta_{F}$ & 0.9 & 0.9 & 0.1 & 0.7 & 0.1 \\
$\mathrm{H} \gamma_{F}$ & 0.8 & 0.8 & 0.2 & 0.7 & 0.1 \\
\hline & & & & &
\end{tabular}

Table 2. "Neighbouring models" in terms of metallicity for $Z=0.02$ and $Z=0.0004$ (top) and in terms of age for the zero points $12 \mathrm{Gyr}$ and $4 \mathrm{Gyr}$ (bottom) used to compute the metallicity sensitivities given in Table 1. Brackets give the corresponding $\Delta Z$ and $\Delta$ age.

\begin{tabular}{llll}
\hline \hline \multicolumn{2}{c}{$Z=0.02$} & \multicolumn{2}{c}{$Z=0.0004$} \\
\hline $0.008(0.012)$ & $0.05(0.03)$ & $0.0001(0.0003)$ & $0.004(0.0036)$ \\
\hline
\end{tabular}

\begin{tabular}{cccc}
\hline \hline \multicolumn{2}{c}{$12 \mathrm{Gyr}$} & \multicolumn{2}{c}{$4 \mathrm{Gyr}$} \\
\hline $11.0(1.0)$ & $13.2(1.2)$ & $3.2(0.8)$ & $5.0(1.0)$ \\
$10.5(1.5)$ & $13.8(1.8)$ & $2.5(1.5)$ & $6.3(2.3)$ \\
\hline
\end{tabular}

Contrary to our expectations, however, at solar metallicity the age-sensitivity of Lick indices is only slightly higher for an intermediate age model compared to the 12 Gyr model; for lowmetallicity SSPs, the effect is more pronounced. Most important, we find that for models at low metallicity, indices are generally much more age-sensitive than for models at high metallicity, especially for age-sensitive indices like G4300 or Balmer line indices. This means that the indices of old, low metallicity GCs can be more sensitive to age than indices of GCs with high metallicity and intermediate age. This is of special interest for any analysis of GC systems involving intermediate age GCs (e.g., in merger remnants), since secondary GC populations with intermediate ages are generally expected to have higher metallicities than "normal" old and metal-poor populations.

Given the limited accuracy of any index measurement in practice, the usefulness of an index to determine age or metallicity depends not only on the relative change in index strength for changing $Z$ or age as it is given by $S$ but also on the absolute change in index strength.

Therefore, in Fig. 3 we show the absolute differences of index strengths for old (12 Gyr) and young (2 Gyr) SSPs for changing metallicity and for metal-rich $([\mathrm{Fe} / \mathrm{H}]=0)$ and metal-poor $([\mathrm{Fe} / \mathrm{H}]=-1.7)$ SSPs for changing age, respectively. Generally, the absolute differences between 8 and 4 Gyr old SSPs are larger than the differences between 8 and 12 Gyr old SSPs at fixed metallicity, as expected (Fig. 3, lower panels). However, this effect is much stronger at low than at high metallicity, which confirms what we get from the $S$ parameter. The absolute differences between models with different metallicity (top panels in Fig. 3) are slighty larger for old than for young SSPs. Interestingly, the plots show that indices known to be sensitive to age can also be highly variable for differing metallicities; especially the broad Balmer indices $\mathrm{H} \delta_{A}$ and $H \gamma_{A}$ change a lot with metallicity. Most important, however, the plot shows that, in practice, moderately metal-sensitive indices like $\mathrm{Mg} b$ can be much more useful for metallicity determinations than indices like Fe5709 or Fe5782, though the latter are, according to the $S$ parameter, much more metal-sensitive.

In order to determine ages and metallicities of GCs, indices should be chosen not only according to known sensitivities as given by $S$, but also according the achievable measurement accuracy and, if possible, according to the expected age and metallicity range of the sources.

\section{The Lick index analysis tool}

Since in the original models (cf. Sect. 2.1), the steps in metallicity are large, in a first step we linearly interpolate in $[\mathrm{Fe} / \mathrm{H}]$ between the 6 metallicities before we analyse any data with our new tool. This is done in steps of $[\mathrm{Fe} / \mathrm{H}]=0.1 \mathrm{dex}$, so the final input grid for the analysis algorithm consists of sets of all 25 Lick indices each for 28 metallicities $(-2.3 \leq[\mathrm{Fe} / \mathrm{H}] \leq+0.4)$ and 4000 ages ( $4 \mathrm{Myr} \leq$ age $\leq 16 \mathrm{Gyr}$ ). Although this approach is only an approximation, the results shown in Sect. 4.2 prove it to be sufficiently accurate.

\subsection{The $\chi^{2}$-approach}

The algorithm is based on the SED analysis tool presented by Anders et al. (2004). The reader is referred to this paper for additional information about the algorithm, as well as for extensive tests using broad-band colours instead of indices.

All observed cluster indices at once - or an arbitrary subsample of them - are compared with the models by assigning a probability $p(n)$ to each model grid point (i.e., to each set of 25 indices defined by 1 age and 1 metallicity)

$p(n) \propto\left(-\chi^{2}\right)$

where

$\chi^{2}=\sum_{i=1}^{25} \frac{\left(I_{\mathrm{obs}}-I_{\text {model }}\right)^{2}}{\sigma_{\mathrm{obs}}^{2}+\sigma_{\mathrm{model}}^{2}}$

with $I_{\mathrm{obs}}$ and $I_{\text {model }}$ the observed and the model indices, respectively, and $\sigma_{\text {obs }}$ and $\sigma_{\text {model }}$ the respective uncertainties. Indices measured in magnitudes are transformed into Ångström before calculation. After normalization $\left(\sum p(n)=1\right)$, the grid point with the highest probability is assumed to be the best model, i.e. it gives the "best age" and the "best metallicity" for the observed cluster.

The uncertainties of the best model in terms of $\pm 1 \sigma$ confidence intervals are computed by rearranging the model grid points by order of decreasing probabilities, and summing up 

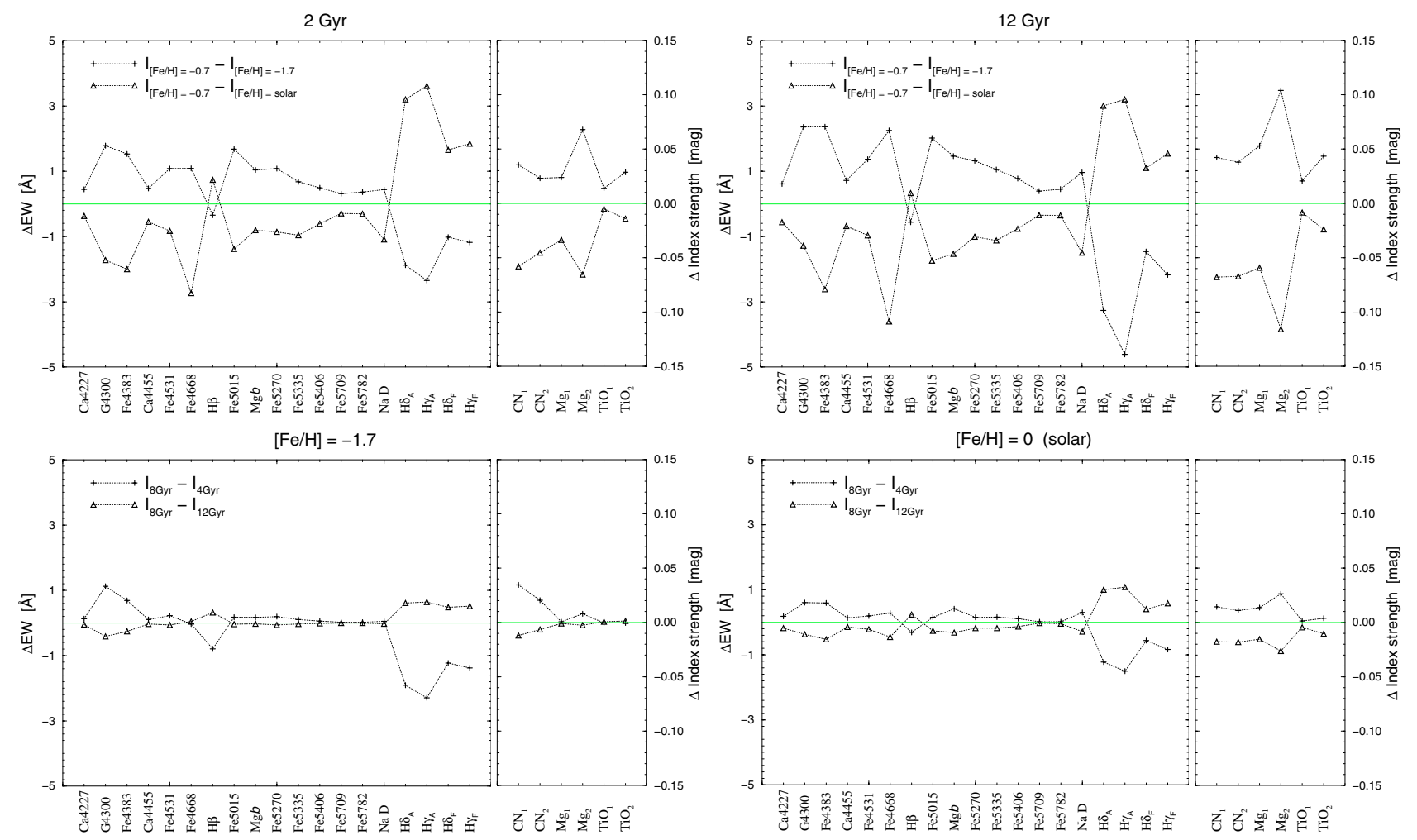

Fig. 3. Absolute differences of index strengths for old and young SSP models for changing metallicity (top), and for metal-rich and metal-poor SSP models for changing age (bottom). The dotted lines are just for presentation.

their probabilities until $\sum p(n)=0.68$ is reached; the $1 \sigma$ uncertainties in age and metallicity are computed from the age and metallicity differences, respectively, of the $n\left(p_{0.68}\right)$ - and the $n\left(p_{\max }\right)$-model. Note that the determination does not take into account the possible existence of several solution "islands" for one cluster; thus the confidence intervals are in fact upper limits.

\subsection{Examples and tests I: Galactic GCs}

We have tested our Lick index analysis tool using a large set of Galactic GCs for which index measurements (taken from Burstein et al. 1984; Covino et al. 1995; Trager et al. $1998^{4}$, and Beasley et al. 2004), as well as age and metallicity determinations from CMD analyses (taken from Salaris \& Weiss 2002) are available.

Figure 4 compares ages and metallicities from both methods. Here, we use the complete set of measured indices available (cf. Table 3) as input for our analysis tool; for comparison, Fig. 5 shows our results using two subsets of indices: the agesensitive indices $\mathrm{Ca} 4227, \mathrm{G} 4300, \mathrm{H} \beta$, and $\mathrm{TiO}_{1}$ in the left panel, and metal-sensitive indices $\mathrm{Mg}_{1}, \mathrm{NaD},[\mathrm{MgFe}]$, plus the agesensitive index $\mathrm{H} \beta$ in the right panel ${ }^{5}$. In all plots, only results with confidence intervals of $\sigma($ age $) \leq 5$ Gyr are plotted ${ }^{6}$.

${ }^{4}$ In this dataset, $\mathrm{H} \delta_{A}, \mathrm{H} \gamma_{A}, \mathrm{H} \delta_{F}$, and $\mathrm{H} \gamma_{F}$ are taken from Kuntschner et al. (2002) who reanalysed the Trager et al. spectra; in the following, "Trager et al. (1998)" always is meant to include this additional data.

$5[\mathrm{MgFe}]$ is a combination of metal-sensitive indices that is known to be widely unaffected by non-solar abundance ratios (see, e.g., Thomas et al. 2003). It is defined as $[\mathrm{MgFe}]:=\sqrt{\langle\mathrm{Fe}\rangle \times \mathrm{Mg} b}$, with $\langle\mathrm{Fe}\rangle:=$ $(\mathrm{Fe} 5270+\mathrm{Fe} 5335) / 2$.

${ }^{6}$ In most cases, very large $1 \sigma$ uncertainties are due to the presence of two "solution islands" (e.g., solution 1: low or intermediate age, solution 2: high age), which are both within their $1 \sigma$ ranges. Since we
The agreement between $[\mathrm{Fe} / \mathrm{H}]$ obtained from our Lick index analysis tool and the corresponding values from CMD analyses is very good, with $\Delta[\mathrm{Fe} / \mathrm{H}] \leq 0.3$ dex when using all available indices, and $\Delta[\mathrm{Fe} / \mathrm{H}] \leq 0.2$ dex when using mainly metal-sensitive indices. With one exception, the age determinations are relatively homogeneous, though the mean age obtained from index analyses is about 2 Gyr too high compared to the results from CMD analyses. Table 4 gives the mean ages and standard deviations of clusters determined using the Lick index analysis tool and from CMD analyses, respectively. It shows that, using all available indices, not only the mean ages but also the age spreads are too high; most likely, this is due to varying horizontal branch (HB) morphologies (see below). However, if only age-sensitive indices are used, the age spread is of the same magnitude than that obtained by CMD analyses.

As an example, Fig. 6 (left) shows the "best-fitting" model for the Galactic GC M3 (NGC 5272), together with the index measurements of Trager et al. (1998) used for the analysis. The best model has an age of $12.88\left(\begin{array}{c}-1.99 \\ +1.75\end{array}\right)$ Gyr and a metallicity of $[\mathrm{Fe} / \mathrm{H}]=-1.7( \pm 0)$ dex; compared with an age of $12.1( \pm 0.7) \mathrm{Gyr}$ and $[\mathrm{Fe} / \mathrm{H}]=-1.66$ dex given by CMD analysis, this is a very good solution. We also give the $\pm 1 \sigma$ confidence intervals of our best model in terms of index values for SSPs with age $12.89-1.99=10.90 \mathrm{Gyr}$ and $12.89+1.74=14.63 \mathrm{Gyr}$, respectively, and metallicity $[\mathrm{Fe} / \mathrm{H}]=-1.7$.

As seen in Figs. 4 and 5, most Galactic GCs are recovered very well in their metallicities by our Lick index analysis tool, in particular when the analysis is concentrated on the set of metalsensitive indices $\mathrm{Mg}_{1}, \mathrm{NaD},[\mathrm{MgFe}]$, plus age-sensitive index $\mathrm{H} \beta$. The origin of the $\sim 2$ Gyr systematic difference between

do not want to use any a priori information about the clusters, we cannot decide between the two solutions and therefore rather omit them completely. 

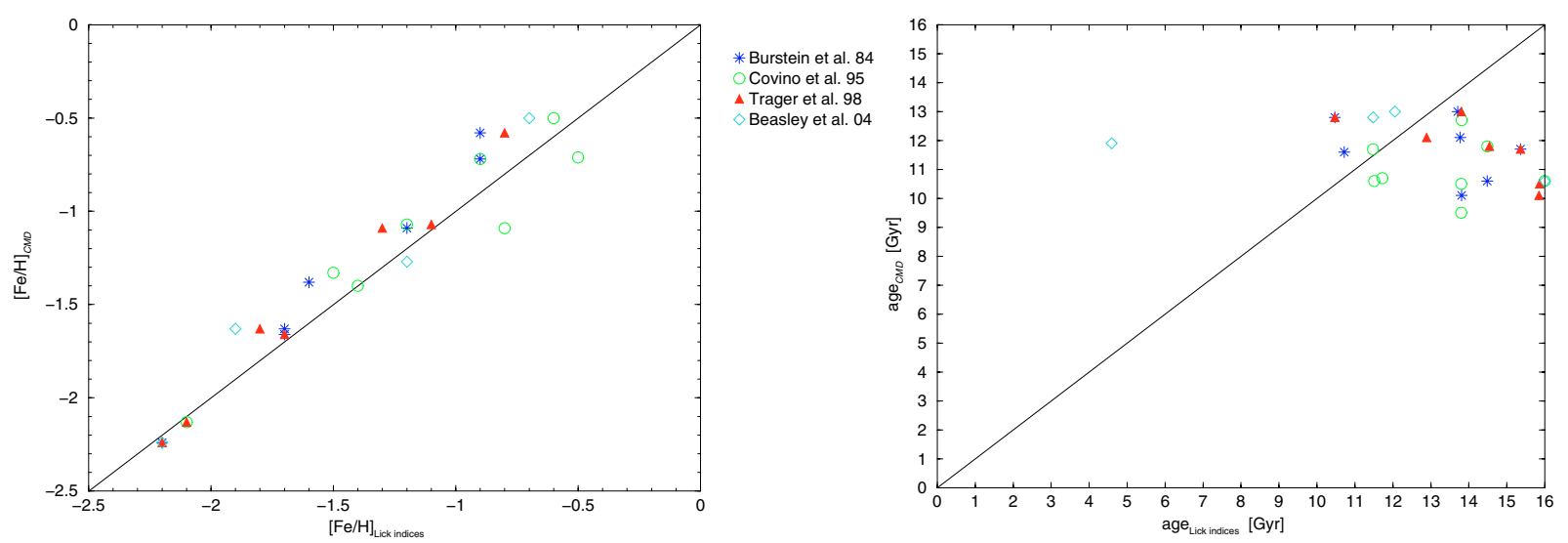

Fig. 4. Galactic GC observations: metallicities (left) and ages (right) determined using our Lick index analysis tool ( $x$-axis, using all measured indices available) vs. metallicities and ages determined by CMD analyses ( $y$-axis, taken from Salaris \& Weiss 2002). Note that only results with model uncertainties of $\sigma(\mathrm{age}) \leq 5 \mathrm{Gyr}$ are plotted.

Table 3. Observations by Burstein et al. (1984, B84), Covinio et al. (1995, C95), Trager et al. (1998, T98; $\mathrm{H} \delta, \mathrm{H} \gamma$ are taken from Kuntschner et al. 2002, see text), Beasley et al. (2004, B04) used to perform the tests of Sect. 4.2. “*”: Index observed, "o": only a subsample of clusters is observed in this index.

\begin{tabular}{|c|c|c|c|c|}
\hline & B84 & C95 & T98 & B04 \\
\hline $\mathrm{CN}_{1}$ & * & o & * & $\mathrm{o}$ \\
\hline $\mathrm{CN}_{2}$ & & & * & o \\
\hline $\mathrm{Ca} 4227$ & & & * & o \\
\hline G4300 & * & o & * & o \\
\hline $\mathrm{Fe} 4383$ & & & o & o \\
\hline Ca4455 & & & $*$ & o \\
\hline $\mathrm{Fe} 4531$ & & & * & o \\
\hline $\mathrm{Fe} 4668$ & & & * & $*$ \\
\hline $\mathrm{H} \beta$ & $*$ & $*$ & $*$ & $*$ \\
\hline Fe5015 & & & * & $*$ \\
\hline $\mathrm{Mg}_{1}$ & * & * & * & $*$ \\
\hline $\mathrm{Mg}_{2}$ & $*$ & $*$ & * & $*$ \\
\hline $\operatorname{Mg} b$ & * & * & $*$ & $*$ \\
\hline Fe5270 & $*$ & * & $*$ & $*$ \\
\hline Fe5335 & $*$ & $*$ & $*$ & $*$ \\
\hline $\mathrm{Fe} 5406$ & & & * & $*$ \\
\hline $\mathrm{Fe} 5709$ & & & $*$ & $*$ \\
\hline Fe5782 & & & $\mathrm{o}$ & $*$ \\
\hline $\mathrm{Na} \mathrm{D}$ & * & o & $*$ & $*$ \\
\hline $\mathrm{TiO}_{1}$ & $*$ & & $*$ & $*$ \\
\hline $\mathrm{TiO}_{2}$ & o & & o & \\
\hline $\mathrm{H} \delta_{A}$ & & & * & $\mathrm{o}$ \\
\hline $\mathrm{H} \gamma_{A}$ & & & o & o \\
\hline $\mathrm{H} \delta_{F}$ & & & * & o \\
\hline $\mathrm{H} \gamma_{F}$ & & & $*$ & o \\
\hline
\end{tabular}

index-determined and CMD-based ages, as well as of the wider age spread we find is, most likely, due to the HB morphologies of the clusters. The Padova isochrones we use for the analyses have very red HBs over most of the parameter space; they have blue $\mathrm{HBs}$ only for metallicities $[\mathrm{Fe} / \mathrm{H}] \leq-1.7$ and ages higher than about 12 Gyr. Therefore, the age of an observed cluster with blue HB can possibly be underestimated by several Gyrs. Proctor et al. (2004), who use a similar technique to the one applied here, also find ages too high compared to values from CMD analyses; depending on the applied SSP models, they find mean ages of 13.1 $( \pm 2.3), 12.2( \pm 3.3)$, and 12.7( \pm 1.9$)$ Gyr, respectively (cf. Table 4). We plan to analyse the influence of HB morphology on Lick index-based age determinations in a separate paper.
Interestingly, and despite the fact that the Lick index measurements used here have very different ages and qualities, the results are of comparable quality for each data set. E.g., the indices taken from Trager et al. (1998) are measured using the same original Lick-spectra as the Burstein et al. (1984) data set; however, the spectra were recalibrated, and more indices were measured. Nonetheless, the results from both data sets are comparable.

Though most results are acceptable, one cluster of our set is seriously misdetermined in terms of age. For the Galactic GC M4 (NGC 6121) the Lick index analysis tool gives an age of only $\sim 5$ Gyr (with a $1 \sigma$ uncertainty of less than 1 Gyr) using both all and only age-sensitive indices, while CMD analysis gives more than twice the age. Since the cluster does not have a very blue HB (Harris 1996, gives an HB ratio of nearly zero), we do not have a reasonable explanation for this. However, anomalies have been found for this cluster, and some properties are still being discussed in the literature (see, e.g., Richer et al. 2004, and references therein). Figure 6 (right) shows models for this "misdetermined" cluster: together with the index measurements taken from Beasley et al. (2004), we show the index values for our best model (i.e., indices for a SSP with age $=4.59\left(\begin{array}{c}-0.31 \\ +0.80\end{array}\right) \mathrm{Gyr}$ and $[\mathrm{Fe} / \mathrm{H}]=-1.2\left(\begin{array}{c}-0.1 \\ +0.0\end{array}\right)$ dex $)$, as well as for a model SSP using the Salaris \& Weiss (2002) solution (age $=11.9( \pm 1.1) \mathrm{Gyr}$ and $[\mathrm{Fe} / \mathrm{H}]=-1.3 \mathrm{dex})$. The indices that differ most between the two models (and for which measurements are available) are $\mathrm{G} 4300, \mathrm{Fe} 4383$, and the Balmer line indices $\mathrm{H} \beta$ and $\mathrm{H} \gamma$; remarkably, the Balmer lines seem to be completely responsible for the misdetermination.

\subsection{Examples and tests II: M31 GCs and non-solar abundance ratios}

Since for Andromeda galaxy (M 31) GCs it is, unfortunately, not possible to obtain high-quality colour magnitude diagrams, reliable determinations of age and metallicity that could be used as "default values" for comparisions are not available. Therefore, for M 31 GCs we can only compare our Lick index-based determinations with results taken from the literature, which are based on spectral indices themselves.

For our analyses, we use the Lick index measurements of M31 GCs presented by Beasley et al. (2004); while not presenting their own age or metallicity determinations for individual clusters, they distinguish four classes for their sample of 

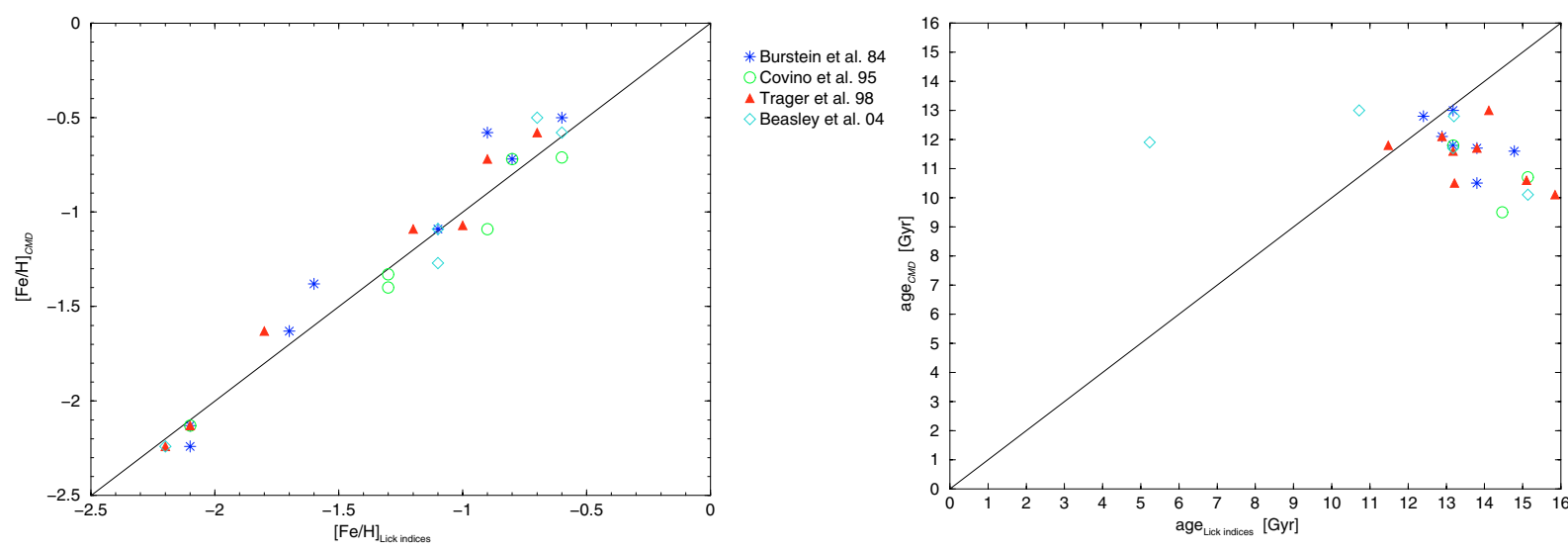

Fig. 5. Same as Fig. 4, but using metallicity-sensitive indices $\mathrm{Mg}_{1}, \mathrm{NaD},[\mathrm{MgFe}]$, plus age-sensitive index $\mathrm{H} \beta$ as input only (left), and using age-sensitive indices $\mathrm{Ca} 4227, \mathrm{G} 4300, \mathrm{H} \beta$, and $\mathrm{TiO}_{1}$ as input only (right).
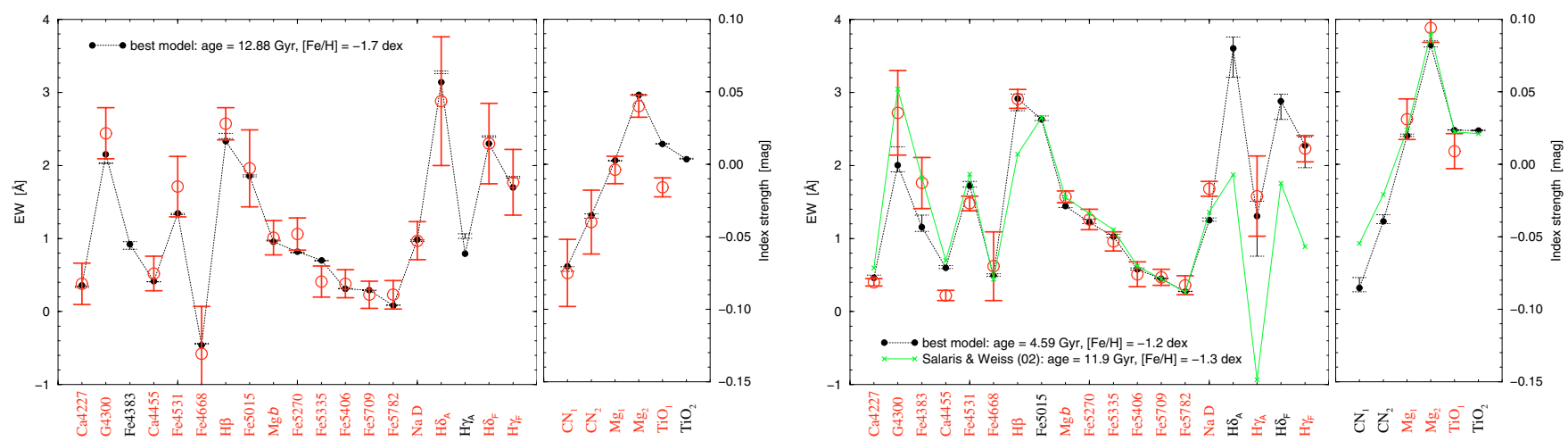

Fig. 6. Left: Lick index measurements of the Galactic GC M3 (NGC 5272) by Trager et al. (1998) with observational errors (open circles) and "best model" indices with the $\pm 1 \sigma$ confidence intervals (black dots). The best model has an age of $12.88\left(\begin{array}{l}-1.99 \\ +1.75\end{array}\right) \mathrm{Gyr}$ and $[\mathrm{Fe} / \mathrm{H}]=-1.7( \pm 0)$ dex; Salaris \& Weiss (2002) give age $=12.1( \pm 0.7) \mathrm{Gyr}$ and $[\mathrm{Fe} / \mathrm{H}]=-1.66$ dex. Right: Lick index measurements of the Galactic GC M 4 (NGC 6121 ) by Beasley et al. (2004) with observational errors (open circles), and "best model" indices with the $\pm 1 \sigma$ confidence intervals (black dots). The best model has an age of only $4.59\left(\begin{array}{c}-0.31 \\ +0.80\end{array}\right) \mathrm{Gyr}$ and $[\mathrm{Fe} / \mathrm{H}]=-1.2\left(\begin{array}{c}-0.1 \\ +00\end{array}\right)$ dex; Salaris \& Weiss $(2002)$ give age $=11.9( \pm 1.1) \mathrm{Gyr}$ and $[\mathrm{Fe} / \mathrm{H}]=-1.27 \mathrm{dex}$. Additionally, we plot model indices for the Salaris \& Weiss (2002) solution, i.e., an $11.9 \mathrm{Gyr} /[\mathrm{Fe} / \mathrm{H}]=-1.3$ dex SSP model (small crosses).

Table 4. Mean ages and standard deviations of cluster ages determined using the Lick index analysis tool and CMD analysis (Salaris \& Weiss 2002), respectively, as shown in Figs. 4 and 5. Note that the values are computed without cluster NGC 6121.

\begin{tabular}{ccccc}
\hline \hline & \multicolumn{2}{c}{ All indices } & \multicolumn{2}{c}{ Age-sensitive indices } \\
& $\langle$ age $\rangle$ & \pm & $\langle$ age $\rangle$ & \pm \\
\hline Lick-analysis & 13.49 & 1.80 & 13.58 & 1.22 \\
CMD-analysis & 11.54 & 1.08 & 11.57 & 1.04 \\
\hline
\end{tabular}

cluster candidates: Young, intermediate age, and "normal" old GCs. Additionally, some sources are suspected to be foreground galaxies. Beasley et al. have measured all available Lick indices with the exception of $\mathrm{TiO}_{2}$.

In Fig. 7, we compare our metallicity determinations using the Lick index analysis tool with results presented by Barmby et al. (2000) (top left panel) and Puzia et al. (2005) (top right panel). While Barmby et al. use calibrations given by Brodie \& Huchra (1990) for their spectroscopic metallicity determinations, using their own measurements of absorption line indices, Puzia et al. (2005) use an $\chi^{2}$ approach using Lick index models from Thomas et al. $(2003,2004)$, which account for nonsolar abundance ratios. Puzia et al. use the same database as we do (i.e., the Lick index measurements published by Beasley et al. 2004). Instead of $[\mathrm{Fe} / \mathrm{H}]$, they give total metallicities $[\mathrm{Z} / \mathrm{H}]$; however, according to Thomas et al. (2003), $[\mathrm{Fe} / \mathrm{H}]$ in the ZW84 scale is in excellent agreement with $[\mathrm{Z} / \mathrm{H}]$. Hence, our results, given in $[\mathrm{Fe} / \mathrm{H}]$, are perfectly comparable to the Puzia et al. results and are appropriate for testing for the influence of non-solar abundance ratios in our results. For both the Barmby et al. (2000) and Puzia et al. (2005) metallicity determinations, we find good agreement with our results. Only for clusters that are classified as young (i.e., age $\leq 1-2$ Gyr) do we find relatively large differences in $[\mathrm{Fe} / \mathrm{H}]$; however, this reflects our expectations, since the models are calibrated using mainly intermediateage or old Galactic stars.

In the bottom panel of Fig. 7, we compare our results with ages determined by Puzia et al. (2005). Again, the results are in surprisingly good agreement, if sources suspected of being foreground dwarf galaxies are not considered. For the set of intermediate-age clusters identified by Beasley et al. (2004), our results reflect this classification perfectly.

Compared with the classification of Beasley et al. (2004), the largest disagreements in both age and metallicity occur for young clusters and for suspected dwarf galaxies; it is no surprise that models computed to fit GCs are not appropriate to galaxies (and, therefore, different methods lead to different results). 

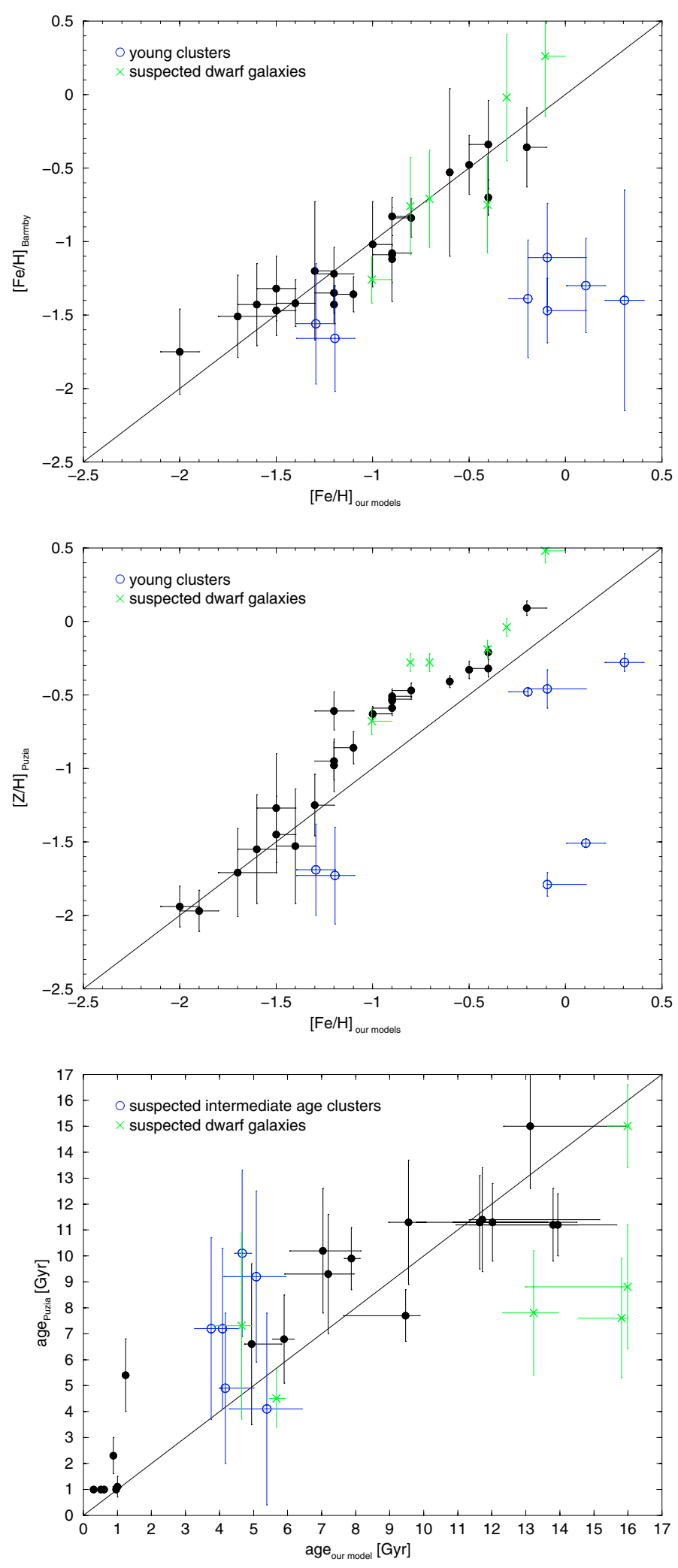

Fig. 7. M 31 GCs: metallicities and ages for the Beasley et al. (2004) GC sample, determined using our Lick index analysis tool ( $x$-axis, using all measured indices available) vs. metallicity determinations taken from Barmby et al. (2000) (top panel) and Puzia et al. (2005) (middle panel), and vs. age determinations taken from Puzia et al. (2005) (bottom panel). The classification as "young cluster" and "suspected dwarf galaxy" is taken from Beasley et al. (2004). See the electronic edition for colour versions of these plots.
Since Puzia et al. (2005) also determine $\alpha$-enhancements for the GC sample, we can check for possible systematic offsets of our determinations compared to theirs due to non-solar abundance ratios.

In Fig. 8, absolute differences between metallicities (left panel) and ages (right panel) derived using our models and from Puzia et al. (2005) are plotted against $[\alpha / \mathrm{Fe}]$. Relatively surprising is that no general trend for the differences in both age and metallicity determinations with $\alpha$-enhancement can be observed, if the large error bars of the $[\alpha / \mathrm{Fe}]$ determinations are taken into account. Hence, the slight offset between metallicities determined by Puzia et al. and by us (cf. Fig. 7, top right panel) for $[\mathrm{Fe} / \mathrm{H}]$ larger than $\sim-1.2$ dex seems not to be due to the use of solar-scaled against $\alpha$-enhanced models.

\section{Summary and outlook}

To cope with the observational progress that makes star cluster $\&$ globular cluster spectra accessible in a wide variety of external galaxies, we have computed a large grid of evolutionary synthesis models for simple stellar populations, including $25 \mathrm{Lick} / \mathrm{IDS}$ indices using the empirical calibrations of Worthey et al. (1994) and Worthey \& Ottaviani (1997). Comparison of the SSP models with Galactic GC observations shows good agreement between models and data.

We find that the well-known and widely used age-sensitive indices $\mathrm{H} \delta_{A}$ and $\mathrm{H} \gamma_{A}$ also show a strong metallicity dependence. The "metallicity sensitivity parameter" $S$ introduced by Worthey (1994) for old stellar populations with solar metallicity is reproduced well by our models. Our models allow us to extend this concept to younger ages and non-solar metallicities. We find the sensitivity of different indices with respect to age and metallicity to depend on age and metallicity; e.g., all indices are generally more age sensitive at low than at high metallicity. Another important issue is the absolute difference in index strength for varying age or metallicity: Due to the limited accuracy of any index measurement, these absolute differences in practice can be of greater importance than the sensitivity given by $S$.

We present a new advanced tool for interpreting absorptionline indices, the Lick index analysis tool LINO. Following an $\chi^{2}$-approach, this tool determines age and metallicity, including their respective $\pm 1 \sigma$ uncertainties, using all, or any subset of, measured indices. Testing our tool against index measurements from various authors for Galactic GCs, which have reliable age and metallicity determinations from CMD analyses in the literature, shows very good agreement: Metallicities of GCs are recovered to \pm 0.2 dex using 6 appropriate indices alone $\left(\mathrm{Mg}_{1}, \mathrm{Mg} b\right.$, $\mathrm{Fe} 5270, \mathrm{Fe} 5335, \mathrm{NaD}, \mathrm{H} \beta$ ). Age determinations from Lick indices consistently yield ages $\sim 2$ Gyr higher than those obtained from CMDs. The origin of this discrepancy is not yet understood. Index measurements for M 31 clusters are analysed and compared to results from the literature, and a good agreement between our results and age and metallicity determinations from the literature is found. We show that the drawback of not having non-solar abundance ratio models does not seriously affect our results.

We will apply $L I N O$ to the interpretation of intermediate-age and old GC populations in external galaxies, complementing our SED analysis tool for the interpretation of broad-band spectral energy distributions. 

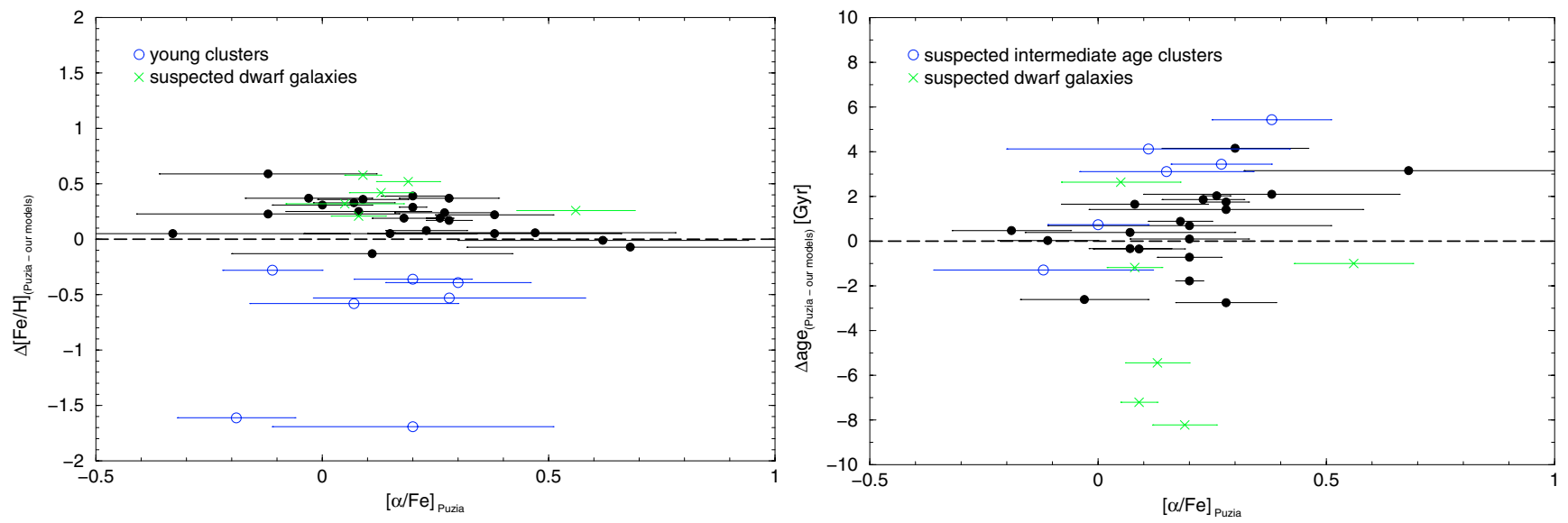

Fig. 8. M 31 GCs: absolute differences between parameters derived using our Lick index analysis tool and results from Puzia et al. (2005), against $[\alpha / \mathrm{Fe}]$ taken from Puzia et al. (2005). Left: $\left([\mathrm{Z} / \mathrm{H}]_{\text {Puzia }}-[\mathrm{Fe} / \mathrm{H}]_{\text {ourmodels }}\right)$; right: $\left(\right.$ age $_{\text {Puzia }}-$ age $\left._{\text {ourmodels }}\right)$. The classification as "intermediate-age cluster" and "suspected dwarf galaxy" is taken from Beasley et al. (2004).

All models are accessible from our website, http://www. astro.physik. uni-goettingen.de/ galev/

Acknowledgements. T.L. is partially supported by DFG grant Fr 916/11-1-2-3.

\section{References}

Anders, P., Fritze, - v., \& Alvensleben, U. 2003, A\&A, 401, 1063

Anders, P., Bissantz, N., Fritze, - v., Alvensleben, U., \& de Grijs, R. 2004, MNRAS, 347, 196

Barmby, P., Huchra, J. P., Brodie, J. P., et al. 2000, AJ, 119, 727

Beasley, M. A., Brodie, J. P., Strader, J., et al. 2004, AJ, 128, 1623

Bertelli, G., Bressan, A., Chiosi, C., Fagotto, F., \& Nasi, E. 1994, A\&AS, 106, 275

Bicker, J., Fritze, - v., Alvensleben, U., Möller, C. S., \& Fricke, K. J. 2004, A\&A, 413,37

Brodie, J. P., \& Huchra, J. P. 1990, ApJ, 362, 503

Burstein, D., Faber, S. M., Gaskell, C. M., \& Krumm, N. 1984, ApJ, 287, 586

Chabrier, G., \& Baraffe, I. 1997, A\&A, 327, 1039

Covino, S., Galletti, S., \& Pasinetti, L. E. 1995, A\&A, 303, 79

Edvardsson, B., Andersen, J., Gustafsson, B., et al. 1993, A\&A, 275, 101

Girardi, L., Bressan, A., Chiosi, C., Bertelli, G., \& Nasi, E. 1996, A\&AS, 117, 113

Gorgas, J., Cardiel, N., Pedraz, S., \& González, J. J. 1999, A\&AS, 139, 29

Harris, W. E. 1996, AJ, 112, 1487

Harris, G. L. H., Harris, W. E., \& Pool, G. B. 1999, AJ, 117, 855

Harris, G. L. H., \& Harris, W. E. 2000, AJ, 120, 2423

Harris, W. E., \& Harris, G. L. H. 2002, AJ, 123, 3108

Kim, Y.-C., Demarque, P., Yi, S. K., \& Alexander, D. R. 2002, ApJS, 143, 499

Kissler-Patig, M. 2000, RvMA, 13, 13
Kuntschner, H., Ziegler, B. L., Sharples, R. M., Worthey, G., \& Fricke, K. J. 2002, A\&A, 395, 761

Kurth, O. M., Fritze, - v., Alvensleben, U., \& Fricke, K. J. 1999, A\&AS, 138, 19

Lejeune, T., Cuisinier, F., \& Buser, R. 1997, A\&AS, 125, 229

Lejeune, T., Cuisinier, F., \& Buser, R. 1998, A\&AS, 130, 65

Lilly, T. 2003, Master's thesis, University Observatory Göttingen (Germany)

Lilly, T., Fritze, - v., \& Alvensleben, U. 2006, A\&A, in preparation

Maraston, C., Greggio, L., Renzini, A., et al. 2003, A\&A, 400, 823

Pagel, B. E. J., \& Tautvaišiene, G. 1995, MNRAS, 276, 505

Puzia, T. H., Saglia, R. P., Kissler-Patig, M., et al. 2002, A\&A, 395, 45

Puzia, T. H., Perrett, K. M., \& Bridges, T. J. 2005, A\&A, 434, 909

Richer, H. B., Fahlmann, G. G., Brewer, J., et al. 2004, AJ, 127, 2771

Salaris, M., \& Weiss, A. 2002, A\&A, 388, 492

Salasnich, B., Girardi, L., Weiss, A., \& Chiosi, C. 2000, A\&A, 361, 1023

Salpeter, E. E. 1955, ApJ, 121, 161

Schulz, J., Fritze, - v., Alvensleben, U., Möller, C. S., \& Fricke, K. J. 2002, A\&A, 392,1

Thomas, D., \& Maraston, C. 2003, A\&A, 401, 429

Thomas, D., Maraston, C., \& Bender, R. 2003, MNRAS, 339, 897

Thomas, D., Maraston, C., \& Korn, A. 2004, MNRAS, 351, 19

Trager, S. C., Worthey, G., Faber, S. M., Burstein, D., \& González, J. J. 1998, ApJS, 116, 1

Trager, S. C., Faber, S. M., Worthey, G., \& González, J. J. 2000a, AJ, 119, 1645

Trager, S. C., Faber, S. M., Worthey, G., \& González, J. J. 2000b, AJ, 120, 165

Tripicco, M. J., \& Bell, R. A. 1995, AJ, 110, 3035

Worthey, G. 1994, ApJS, 95, 107

Worthey, G., Faber, S. M., González, J. J., \& Burstein, D. 1994, ApJS, 94, 687

Worthey, G., \& Ottaviani, D. L. 1997, ApJS, 111, 377

Worthey, G. 2004, private communication

Zepf, S. 1999, AAS, 194, 4015

Zepf, S. 2002, IAUS, 207, 653 